\title{
La Cartografía de las Unidades Inferiores de la Regionalización Físico-Geográfica (RFG) de Michoacán ${ }^{\text {ț }}$
}

\section{Map of the lower units of the Physical Geographic Regionalization(RFG) for Michoacán.}

\author{
Alejandra Espinoza Maya*a, Manuel Bollo Manent ${ }^{\mathrm{a}}$ \\ ${ }^{a}$ Centro de Investigaciones en Geografía Ambiental, UNAM - Campus Morelia, Morelia, México, C.P. 58190
}

\begin{abstract}
Resumen
El presente trabajo propone la Regionalización físico-geográfica (RFG) para Michoacán. Se basa en el método de distinción de unidades de la regionalización, a partir del mapa de la tipología regional de los paisajes, por medio de la asociación de paisajes de esta tipología en cada nivel taxonómico de la RFG. Se parte de la diferenciación de las Provincias físico-geográficas del territorio como unidad taxonómica superior que es posible representar a la escala de estudio y posteriormente se establecen las unidades inferiores de la taxonomía a su interior. Para Michoacán se distinguieron: 2 Provincias, 7 Subprovincias, 17 Distritos y 36 Regiones físico-geográficas en una superficie de 58296,11 $\mathrm{km}^{2}$, a escala 1:500,000. La delimitación de estas unidades espaciales, resulta útil en diversos ámbitos, particularmente en la gestión, manejo y planeamiento del territorio, ya que están constituidas por niveles jerárquicos de unidades anidadas por medio de una taxonomía que permite abarcar diferentes escalas.
\end{abstract}

Palabras clave: Regionalización; paisaje físico-geográfico; taxonomía.

\begin{abstract}
The present paper presents the Map of the lower units of the Physical Geographic Regionalization (RFG) for Michoacán. It is based on the method of units distinction of the regionalization, based on the map of the regional typology of the landscapes, by means of the association of landscapes of this typology in each taxonomic level of the RFG. It starts from the differentiation of the physicalgeographic Provinces of the territory as a higher taxonomic unit and subsequently establishes the lower units of the taxonomy to its interior. For Michoacán, 2 Provinces, 7 Sub-provinces, 17 Districts and 36 physical-geographic Regions were distinguished on an area of $58296.11 \mathrm{~km}^{2}$, at a scale of 1: 500,000 . The delimitation of these spatial units is useful in several areas, particularly in management and land planning, since they are constituted by hierarchical levels of nested units by means of a taxonomy allowing the coverage of different scales.
\end{abstract}

Keywords: Regionalization; physical-geographical landscape; taxonomy.

\section{Introducción}

Una Regionalización es un sistema de división territorial de unidades espaciales de diferentes tipos (administrativas, econó-

\footnotetext{
(C) Alejandra Espinoza Maya \& Manuel Bollo Manent . Published by Terra Digitalis.

This is an Open Access article distributed under the terms of the Creative Commons Attribution License (https://creativecommons.org/licenses/by-nc-sa/4.0/), which permits non-commencial sharing of the work and adaptions, provided the original work is properly cited and the new creations are licensed under identical terms.
}

*E-mail address: aespinoza@pmip.unam.mx micas o naturales). La Regionalización físico-geográfica (RFG) o de paisajes, permite entender la composición, estructura, relaciones, diferenciación y desarrollo de los paisajes físico geográficos (f-g) individuales (geosistemas, complejos f-g) naturales. El paisaje natural es el resultado de la interacción de los componentes naturales (suelos, relieve, roca, agua clima y organismos vivos) en un espacio dado de la superficie terrestre.

Los principios básicos para la distinción de las unidades de la RFG son el estructural-genético y el histórico-evolutivo y para dicha distinción se requiere de procedimientos de análisis, clasificación y cartografía de estos paisajes, vistos como unida- 
des espaciales con un mismo origen y con integridad territorial; en ellos se desarrollan interacciones entre los componentes naturales que les constituyen y de estas interacciones depende su homogeneidad relativa (Mateo, 2002).

Existen dos vías para la clasificación de los paisajes a nivel regional: la Regionalización y la Tipología físico - geográficas; estas se encuentran íntimamente relacionadas y son parte de los estudios básicos en la Geografía Física, y en particular de la Geografía del Paisaje. Estas formas de clasificación del paisaje se realizan al igual que las de cualquier componente natural, como el suelo, el clima o los aspectos geológicos, tan solo que la clasificación de los paisajes se realiza en un nivel de mayor complejidad al mostrar los resultados de diferenciación espacial de la interrelación entre todos los componentes naturales. El resultado en ambos casos es la diferenciación en unidades espaciales de un territorio a escala regional, según los criterios específicos de cada clasificación, diferenciación que se muestra por medio de la cartografía de sus diferentes niveles taxonómicos. El proceso de clasificación del espacio, en ambas, es el resultado de la integración entre los componentes naturales, pero según criterios diferentes.

La Tipología f-g distingue los paisajes cuando integra los componentes naturales, pero identifica los espacios con rasgos comunes, que son análogos. Los paisajes f-g tipológicos son por tanto repetibles en el espacio y en el tiempo, su clasificación se realiza por analogía en función de determinados rasgos comunes de los componentes que conforman su estructura (Mateo, 2002).

La RFG es un sistema de subdivisión del espacio en el cual se clasifican y delimitan los paisajes f-g por rasgos característicos, propios, únicos de cada porción del espacio. Los paisajes f-g individuales se determinan a partir de los principios de objetividad, integridad territorial, complejidad, homogeneidad, unidad genética y combinación de factores zonales y azonales de la diferenciación geográfica. Estos criterios son el soporte para el diagnóstico y distinción de las unidades de la RFG (Isachenko 1991:222), las que se representan cartográficamente en un sólo areal, con nombres propios. Es un proceso de "personalización" del espacio, lo que hace que cada paisaje f-g de la regionalización sea único en la naturaleza (Bollo et al., 2015).

El objetivo de la RFG de un territorio es establecer un sistema de subdivisión de unidades espaciales (paisajes f-g), con carácter individual e irrepetible, integradas en niveles jerárquicos, anidadas por medio de una taxonomía definida por índices diagnósticos en cada nivel taxonómico y delimitar dichas unidades expresándolas cartográficamente. De aquí, que toda RFG de un territorio contenga el mapa de las unidades espaciales delimitadas para cada nivel taxonómico y un texto que les caracterice y describa.

La RFG, surge en Rusia a partir de los trabajos de V.V. Dokuchaev a finales del siglo XIX; en los inicios del siglo XX se realizaron distintas regionalizaciones aplicando sus principales conceptos y enfoques (Berg, 1913; Suslov-Serguei, 1947). A mitad del siglo XX se comenzó a prestar atención en la RFG también en otros países del ex-bloque socialista, en la Alema- nia Federal, por ejemplo, se comenzó con una detallada RFG del territorio. Estas investigaciones sentaron las bases de las RFGs actuales; entre 1960 y 1987 quedan establecidos los principios básicos y métodos de la RFG (Bollo et al., 2015). Hoy la RFG se realiza en numerosos países del mundo. A partir de 1978 se realizan las primeras RFGs en América Latina. La RFG de América Central y del Caribe (Mateo, 1989), la RFG de Cuba; en Brasil actualmente se realiza la RFG con el uso de una metodología similar (Mateo et al., 2004). En México se han realizado regionalizaciones naturales con enfoques diferentes a la RFG, por ejemplo las Regionalizaciones Fisiográficas de México de Ordóñez (1936, 1946), Álvarez (1961) y Alcorta (1964), la Regionalización Geomorfológica de Michoacán de Ocampo (Bocco et al., 1999). SEDUE (1988) elaboró una Regionalización Ecológica de México de carácter tipológico. Las regionalizaciones posteriores (Cuanalo 1989; Quiñones, 1987; Barajas et al., 1986), también fisiográficas, toman en cuenta solo el relieve y su génesis en la clasificación de las unidades; aunque tienen mayor complejidad en el proceso de sistematización, al igual que las anteriormente descritas, no siguen el enfoque regional en toda la clasificación de unidades y no integran la interrelación de los componentes naturales (Bollo et al., 2015).

Por su parte, la RFG abarca diferentes escalas de análisis ya que está integrada por niveles jerárquicos que abarcan unidades superiores e inferiores, las cuales se encuentran anidadas por medio de una taxonomía. Las unidades en la RFG son únicas e irrepetibles en el espacio, definidas por medio de índices diagnóstico, los cuales establecen los criterios de clasificación para distinguir las unidades en cada nivel taxonómico de la RFG. En comparación con otras regionalizaciones naturales, la RFG permite entender de una forma más organizada la estructura espacial del territorio a través de una precisa identificación, clasificación, caracterización y cartografía de las unidades espaciales individuales en el nivel regional.

La propuesta de RFG de México, realizada por Bollo et al., 2015, a partir de la cual se distinguieron las unidades superiores de la clasificación y se iniciaron los trabajos de distinción de las unidades taxonómicas inferiores en el territorio mexicano, es base del presente trabajo.

La RFG de México, en sus unidades superiores, ha sido publicada en el libro "Una propuesta de Regionalización físicogeográfica de México” (Bollo et al., 2015). En ella, se distinguen tres niveles taxonómicos para dichas unidades superiores; Subcontinente (1 Subcontinente), Países (5), Territorios (10) y las Provincias físico-geográficas (15) como el nivel intermedio entre las unidades superiores, asimismo se presenta la cartografía de estas unidades, así como los índices diagnóstico que las definen. Además se proponen las unidades taxonómicas inferiores en tres niveles taxonómicos: Subprovincias, Distritos y Regiones físico-geográficas; y se establecen los índices diagnósticos que definen a dichas unidades.

Como antecedentes de clasificación, distinción y cartografía de unidades inferiores de la RFG en México, se señalan los trabajos realizados en la Provincia f-g Península de Baja California (Zaragoza, 2010) y de cuatro Subprovincias de la Provincia 
f-g Sierra Madre del Sur, en las que se ubica el estado de Guerrero (Ortíz et al., 2015). También, se trabaja en la propuesta de la RFG en las Subprovincias que contienen al estado de Jalisco, la cual abarca parte de las Provincias f-g Eje Neovolcánico Transmexicano y Sierra Madre Occidental. En consecuencia, y como continuidad de estos dos últimos trabajos mencionados, surge la propuesta de realizar la RFG y su cartografía en sus unidades inferiores para Michoacán en las Provincias f-g antes mencionadas.

El objetivo de este trabajo es presentar el mapa de las unidades taxonómicas inferiores de la RFG sobre las que se ubica el territorio de Michoacán y mostrar la metodología utilizada para establecer y delimitar dichas unidades, así como su importancia para los estudios básicos en la geografía de este territorio y sus posibles aplicaciones.

La delimitación obtenida de las unidades inferiores de la clasificación para el territorio de Michoacán, se realiza con el apoyo de herramientas (SIG) y métodos científicos del análisis espacial y se muestran con ayuda de la cartografía, en el "Mapa de las unidades inferiores de la Regionalización físico - geográfica de Michoacán de Ocampo" (Lámina 1). Las unidades espaciales mostradas en el mapa mencionado, son de utilidad en diagnósticos y evaluaciones ambientales, en el análisis de potenciales naturales y en la evaluación de recursos naturales, en la planificación ambiental y territorial, en la estructuración de planes de desarrollo, entre otros estudios a nivel regional.

La RFG de Michoacán sirve de base a una tesis doctoral, la cual toma como unidades espaciales de análisis a las Regiones físico-geográficas para realizar diferentes evaluaciones ambientales en el territorio.

\section{Metodología}

Existen diferentes métodos para establecer las unidades inferiores de la RFG de un territorio, el más utilizado y aplicado en este trabajo, es un método inductivo que se conoce como el ... método de distinción de los paisajes regionales de la RFG sobre la base del análisis del mapa tipológico regional de los paisajes, el cual parte de dos principios: primero, que la clasificación tipológica regional de los paisajes caracteriza la estructura de la envoltura geográfica a ese nivel por lo que constituye una buena base para la RFG y segundo, que a escala regional, una unidad físico - geográfica individual es el resultado de la asociación definida de paisajes (de carácter tipológico) en cada nivel taxonómico de la RFG... (Bollo et al., 2015:22).

En la tabla 1 se presentan los sistemas de unidades taxonómicas de la RFG y de la Tipología f-g en el nivel regional, más utilizados internacionalmente así como su escala de representación. También se muestran las unidades de la taxonomía de los paisajes del nivel local. La escala 1:250 000 es de transición entre el nivel local y regional. Por la homogeneidad de los paisajes no es posible realizar regionalización a nivel local, donde sólo se puede realizar la clasificación tipológica del paisaje (Bollo et al., 2015).
Las unidades superiores definidas en la RFG de México, en las que se ubica el territorio de Michoacán se presentan en la figura 1. Como se puede observar el territorio de estudio abarca parte de dos Países, dos Territorios y dos Provincias físicogeográficas.

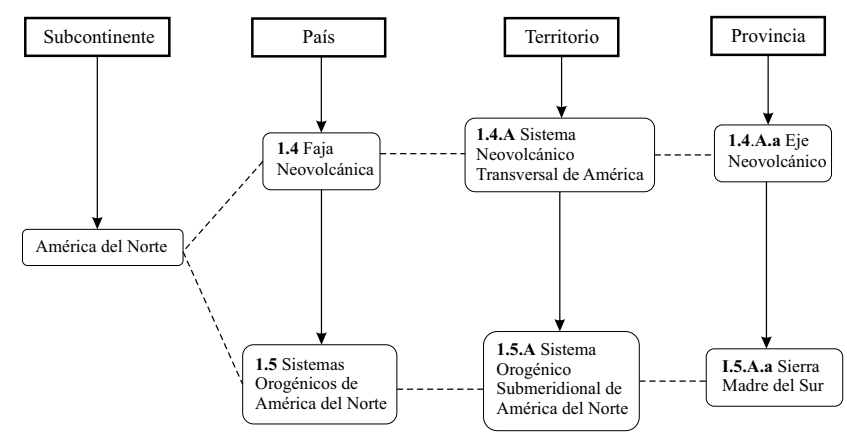

Figura 1: Unidades superiores de la Regionalización Físico Geográfica de México a las que pertenece el estado de Michoacán.

Figure 1: Upper unities of the Physical Geographic Regionalization of Michoacan. /

El proceso metodológico parte de la distinción de las unidades inferiores: Subprovincias, Distritos y Regiones f-g (Figura 2), a partir de las Provincias físico-geográficas, donde se ubica el territorio michoacano y consiste en la aplicación del método inductivo mencionado o de asociación de las unidades de la tipología (según el nivel taxonómico de la clasificación), para delimitar unidades de la RFG.

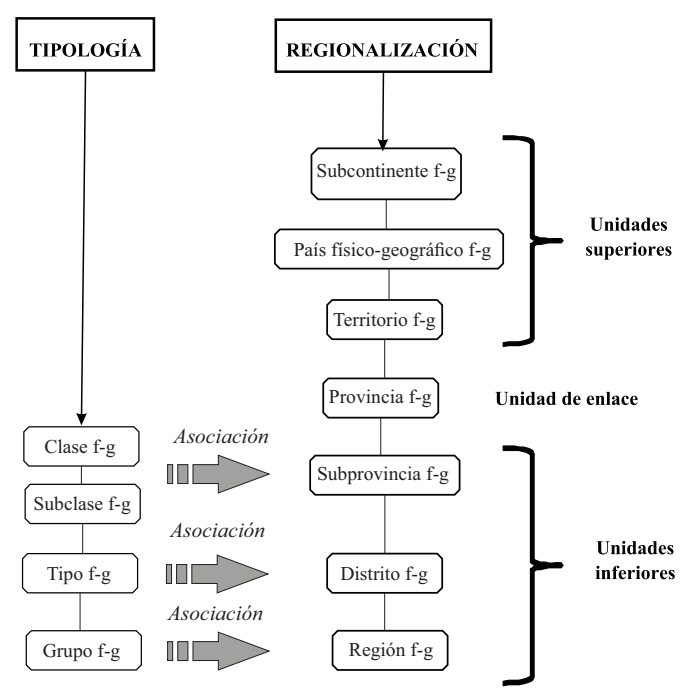

Figura 2: Relación entre las unidades taxonómicas de la Tipología y Regionalización de los paisajes físico - geográficos del nivel Regional. Figure 2: Relationship between the taxonomical unities of the Tipology and the Regionalization of the physical geographic landscapes of Regional level. 
Tabla 1: Unidades taxonómicas más comunes de la cartografía regional en el enfoque físico-geográfico. Table 1: Most common taxonomic unities of the regional cartography, according to the physical-geographic perspective.

\begin{tabular}{|c|c|c|}
\hline \multicolumn{3}{|c|}{$\begin{array}{c}\text { Nomenclatura de las unidades" } \\
\text { Nivel Escala }\end{array}$} \\
\hline & \multirow{3}{*}{$\begin{array}{c}\text { Nivel Local } \\
(\geq 1: 250000) \\
\text { Tipología Local }\end{array}$} \\
\hline \multicolumn{2}{|c|}{$\begin{array}{l}\text { Nivel Regional } \\
(\leq 1: 250000 \text { hasta } \leq 1: 1000000)\end{array}$} & \\
\hline Unidades Individuales (RFG) & $\begin{array}{c}\text { Unidades Tipológicas (Tipología } \\
\text { Regional) }\end{array}$ & \\
\hline Continente & Clase & Localidad \\
\hline Subcontinente & Tipo & Comarca \\
\hline País & Grupo & Subcomarca \\
\hline Territorio & Especie & Facie \\
\hline Provincia & & \\
\hline Subprovincia & & \\
\hline Distrito & & \\
\hline Región & & \\
\hline
\end{tabular}

* (Modificado de Mateo, 1984)

Para ello es necesario además, complementar el análisis espacial con otros índices diagnóstico para cada unidad inferior (Tabla 2).

Para poder establecer la asociación de unidades de la tipología regional que conforman las unidades inferiores de la RFG, fue necesaria la actualización, así como la revisión de los límites de los paisajes del "Mapa de Paisajes físico-geográficos del Estado de Michoacán" (Ramírez et al., 2012) (Tipología regional de los paisajes de Michoacán). Con el apoyo de tecnologías de SIG, y la visualización del Modelo Digital del Terreno (MDT), modelo altitudinal, y el uso de cartografía temática de ríos, cuerpos de agua, cobertura vegetal y morfología del relieve, se rectificaron dichos límites (Información cartográfica digital 2015-2016).

Actualizadas las unidades inferiores de la tipología regional (Clases, Subclases, Tipos y Grupos de paisajes f-g), establecidas las diferentes formas del relieve (tipos morfoestructurales, megaformas, macroformas y mesoformas), así como la tipología del clima (Grupos, Subgrupos, Tipos y Subtipos), se inicia la distinción de las unidades inferiores de la RFG (Tabla 3). Con ayuda del MDT, se establecen los límites sobre el mapa de la tipología regional, utilizando los propios límites de las unidades tipológicas. Se establecen y delimitan las Subprovincias $\mathrm{f}-\mathrm{g}$ al interior de las Provincias f-g, se delimitan los Distritos $\mathrm{f}-\mathrm{g}$ al interior de las Subprovincias y las Regiones $\mathrm{f}-\mathrm{g}$ al interior de los Distritos a partir de los índices diagnóstico que les definen (ver Tabla 2). Es decir, es necesario un proceso de análisis que consiste en la integración, síntesis, revisión, organización, y homologación del espacio para obtener las unidades en cuestión. Una vez establecidos los límites de las unidades de los tres niveles taxonómicos, se obtiene como resultado y objetivo del trabajo, el "Mapa de las unidades inferiores de la Regionalización físico - geográfica de Michoacán de Ocampo". Se confecciona la leyenda y se realiza la edición del mapa final. La figura 3 , muestra las etapas de este proceso metodológico.

En la tabla 3 se muestra un ejemplo del uso de los índices diagnóstico en la conformación y delimitación de las diferentes unidades de la regionalización en el territorio.

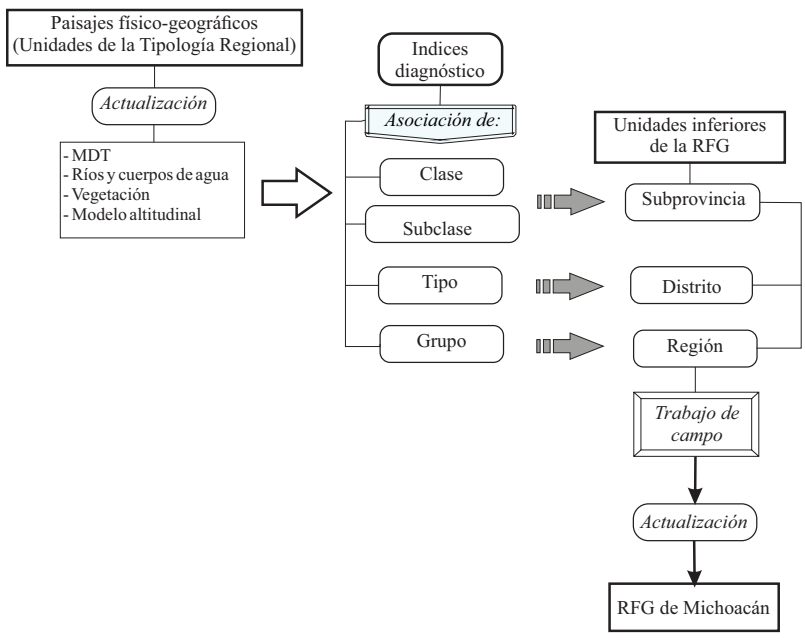

Figura 3: Proceso metodológico para la distinción de las unidades inferiores de la RFG para el estado de Michoacán. / Figure 3 : Metodological process followed to generate the lower unities of the Physical Geographic Regionalization (RFG) of Michoacan

El análisis de dichos índices permitió observar una correspondencia entre las Provincias y Subprovincias f-g con las mismas unidades de la clasificación fisiográfica elaborada por INEGI (Quiñones, 1987), sin embargo los límites de estas unidades de la RFG, al ser establecidos por las Clases y Subclases de paisaje en el mapa tipológico se diferencian al tiempo que son más precisos. Ello se debe a que el indicador que define a las Provincias y Subprovincias fisiográficas, es semejante a uno de los índices diagnóstico que describen a las Provincias y Subprovincias físico-geográficas (morfoestructuras y megaformas del relieve). Ello, como se explica a continuación, tiene relación con la nomenclatura y números de identificación presentados como leyenda del mapa para estos dos niveles taxonómicos.

La tabla 4 muestra el número de la clasificación que le co- 
Tabla 2: Índices diagnóstico de las unidades inferiores de la Regionalización Físico Geográfica de México (Bollo et al. 2015)

Table 2: Diagnostic indexes of the lower unities of the Physical Geographic Regionalization (RFG) of Mexico

\begin{tabular}{|c|c|c|}
\hline \multicolumn{3}{|c|}{ Índices Diagnóstico } \\
\hline Subprovincia & Distrito & Región \\
\hline $\begin{array}{l}\text { 1. Asociación de clases y subclases de Pai- } \\
\text { saje (Grupos y subgrupos de clima). } \\
\text { 2. Predominio de megaformas de relieve } \\
\text { (asociación de tipos morfoestructurales). } \\
\text { 3. Comunidad espacial }\end{array}$ & $\begin{array}{l}\text { 1. Asociación de tipos de Paisaje. (Simila- } \\
\text { res características bioclimáticas) } \\
\text { 2. Asociación de tipos de clima } \\
\text { 3. Asociación de macroformas del relieve } \\
\text { predominio de un tipo morfoestructural } \\
\text { 4. Comunidad espacial }\end{array}$ & $\begin{array}{l}\text { 1. Asociación de grupos de Paisaje. } \\
\text { 2. Asociación de mesoformas del relieve. } \\
\text { 3. Asociación de subtipos de clima. } \\
\text { 4. Comunidad espacial. }\end{array}$ \\
\hline
\end{tabular}

rresponde a cada Provincia y Subprovincia f-g en la leyenda del mapa en cuestión. Para identificar los Distritos f-g en la leyenda se consideran letras mayúsculas y para las Regiones f-g se asignan letras minúsculas. La nomenclatura en la clasificación queda como se muestra en el siguiente ejemplo:

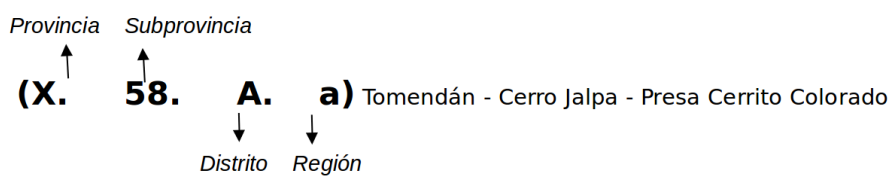

Los nombres de las Provincias y Subprovincias f-g, se relacionan con las morfoestructuras, mega y mesoformas del relieve que les definen. Los nombres de los Distritos y Regiones f-g se establecen a partir de la localidad administrativa de mayor superficie o la localidad administrativa con el mayor número de habitantes, el rasgo orográfico de mayor altitud, y otros rasgos geográficos, hidrológicos y culturales de importancia.

\section{Resultados}

Como resultado del proceso metodológico se distinguieron, delimitaron y representaron cartográficamente 7 Subprovincias, 17 Distritos y 36 Regiones f-g (Lámina 1; Tabla 4). Dada la homogeneidad, resultado de la interrelación de los componentes naturales de algunas partes del territorio, hay Distritos que contienen una sola Región f-g.

La RFG de un territorio está constituida por el mapa de las unidades de la RFG y un texto explicativo que describe y caracteriza a cada unidad. Como explicamos anteriormente, las unidades de la RFG integran los componentes naturales en los espacios que contienen; tales como geología, litología, relieve, clima, cobertura de vegetación, edafología e hidrografía y también unidades sintéticas de la tipología de los paisajes en cada nivel taxonómico, por lo cual y como complemento del mapa, es necesaria una descripción de dichos componentes naturales y de los paisajes tipológicos que integran cada unidad, así como explicar cómo se cumplen los índices diagnóstico que les identifican. Ello se realiza en un texto que caracteriza y describe a cada unidad, el cual es objeto de una publicación en forma de libro que se encuentra en preparación.

La Provincia f-g Eje Neovolcánico Transversal (ENT) ocupa el $46.3 \%$ de la superficie de Michoacán. Esta unidad f-g se integra por 4 Subprovincias, 8 Distritos y 16 Regiones (Tabla 4; Lámina 1). Al interior de la Provincia f-g ENT, la Subprovincia de mayor superficie es la Neovolcánica Tarasca, la cual ocupa un área de $10611.02 \mathrm{~km}^{2}$ (39.3\%) (Figura 4).

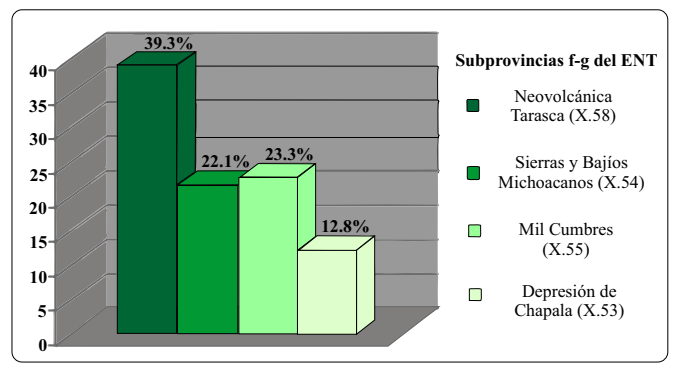

Figura 4: Superficie en porciento que ocupan las Subprovincias físico-geográficas del Eje Neovolcánico Transmexicano en el estado de Michoacán. / Figure 4: Percentage of the surface occupied by the Physical-Geographic Subprovinces of the Eje Neovolcánico Transmexicano in Michoacan.

La Provincia f-g Sierra Madre del Sur (SMS) abarca el $53.7 \%$ de Michoacán. Se integra por 3 Subprovincias, 9 Distritos y 20 Regiones (Tabla 4; Lámina 1). La Subprovincia f-g de mayor extensión territorial es la Cordillera Costera del Sur, que cuenta con $20682.4 \mathrm{~km}^{2}$ (66.1\% de la unidad) (Figura 5).

El Distrito f-g de mayor tamaño al interior de Michoacán es Uruapan - Cerro Tancítaro - Lago de Pátzcuaro (X.58.B) con $7070,82 \mathrm{~km}^{2}$ lo que representa el $12.1 \%$ del Estado; forma parte del ENT y de la Subprovincia f-g Neovolcánica Tarasca (X.58). Debido a la homogeneidad de las características naturales de este Distrito f-g no fue posible su delimitación en dos taxones con características diferentes entre sí, por lo que representa a su vez una Región f-g con las mismas características que dicho Distrito.

La segunda Región f-g por su extensión en Michoacán es 
Tabla 3: Índices diagnóstico aplicados en tres niveles taxonómicos de la RFG (Subprovincia, Distrito y Región), en unidades pertenecientes a la Subprovincia f-g Neovolcánica Tarasca (X.58)

Table 3: Diagnostic indexes, applied in three taxonomical levels (Subprovince, District and Region), to the Neovolcanica Tarasca (X.58) Subprovince.

\begin{tabular}{ccc}
\hline & Índices Diagnóstico & \\
\hline Subprovincia & Distrito & Región \\
\hline
\end{tabular}

1. Asociación de clases y subclases de Paisaje (Grupos y subgrupos de clima).

2. Predominio de megaformas de relieve (asociación de tipos morfoestructurales).

3. Comunidad espacial
1. Asociación de tipos de Paisaje. (Similares características bioclimáticas)

2. Asociación de tipos de clima

3. Asociación de macroformas del relieve predominio de un tipo morfoestructural

4. Comunidad espacial
1. Asociación de grupos de Paisaje.

2. Asociación de mesoformas del relieve.

3. Asociación de subtipos de clima.

4. Comunidad espacial.

\section{Neovolcánica Tarasca $(\mathbf{X . 5 8 )}$ \\ Clase predominante de paisaje $f$-g \\ A2. Montañas, lomeríos, piedemontes y planicies en climas húmedos templados.}

Uruapan - Cerro Tancítaro - Lago de Pátzcuaro (X.58.B)

\section{Subclase de paisajes $f$-g}

A2.IV. Lomeríos volcánicos y tectónicovolcánicos en climas templados.

\section{Megaformas del relieve}

29. Corresponde a Sierras y Bajíos Michoacanos, Bajío Guanajuatense y Depresión de Chapala

30. Corresponde a Llanuras y Sierras de Querétaro e Hidalgo, Mil cumbres, Escarpa Limítrofe del Sur y Sierra Neovolcánica Tarasca

\section{Tipo predominante de paisajes $f$-g}

A2.IV.2. Lomeríos volcánicos y tectónicovolcánicos, en rocas extrusivas básicas, en clima templado, con bosque de coníferas y latifoliadas y áreas no forestales, 1 sobre Andosol y Leptosol.

\section{Macroformas del relieve}

Sierras de Comanja, Quiroga, Nahuatzen, Paracho, Tancítaro, Patamban, Acuitzio, del Tigre y de Tingambato.

\section{Uruapan - Pátzcuaro - Meseta Purépecha}

(X.58.B.a)

Grupo predominante de paisaje $f$-g

A2.IV.2.32. Lomeríos volcánicos constituidos por rocas extrusivas básicas en clima templado húmedo a subhúmedo, con bosque de pino-encino y áreas no forestales, sobre Andosol, Leptosol, y Luvisol.

\section{Mesoformas del relieve}

Lomeríos y montañas, Cerro San Miguel

${ }^{1}$ Áreas donde se ha sustituido la vegetación natural

Arteaga - Cerro El Capulín (XII.66.B.a), con 5130.39 km², el $8.8 \%$ del Estado.

Una vez concluido el mapa, se realizaron recorridos de campo en todo el territorio, se verificaron los límites y se realizó una bitácora fotográfica de todas las Regiones físico-geográficas.

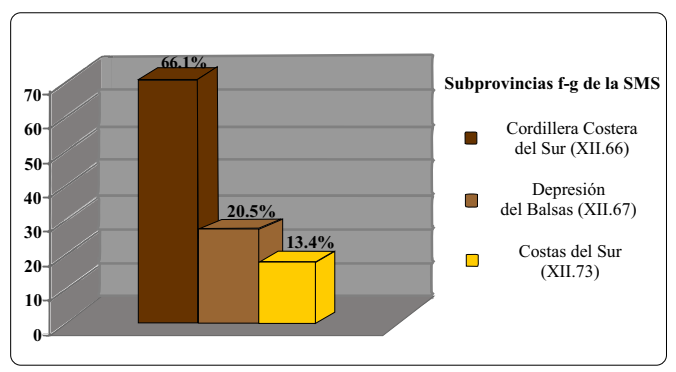

Figura 5: Superficie en porciento que ocupan las Subprovincias físico-geográficas de la Sierra Madre del Sur en el estado de Michoacán. / Figure 5: Percentage of the surface occupied by the Physical-Geographic Subprovinces of the Sierra Madre del Sur in Michoacan.

El "Mapa de las unidades inferiores de la Regionalización físico-geográfica de Michoacán de Ocampo", y su texto des- criptivo, son herramientas útiles y modernas, que permiten la divulgación de un conocimiento geográfico actualizado de la Entidad para muy diversos usuarios y constituyen una caracterización y descripción de la geografía física michoacana y su expresión cartográfica.

A partir de la RFG propuesta, es posible realizar regionalizaciones aplicadas tomando como base a las unidades de la RFG; a partir de la evaluación de sus potenciales naturales para diferentes usos, es posible realizar la regionalización de la actividad agrícola, la turística, la minera, la industrial, entre muchas otras.

La RFG parte del principio de objetividad de sus unidades espaciales, las cuales han constituido el medio biofísico del cual la sociedad se ha apropiado, que habita, y en el cual realiza sus actividades sociales, culturales y económicas, modificándolas. $\mathrm{Si}$ identificamos por medio de indicadores biofísicos, demográficos, culturales, sociales y económicos, cómo ocurren esos procesos en dichos espacios, podremos tratar dichas unidades como sistemas ambientales complejos, resultado de las interacciones entre la Naturaleza y la Sociedad y por tanto realizar diagnósticos y evaluaciones ambientales de carácter holístico, con apoyo del análisis espacial, a diferentes niveles en la escala 
Tabla 4: Unidades inferiores f-g que integran la Regionalización Físico Geográfica de Michoacán de Ocampo (Claves de identificación en la Lámina 1 y superficie en $\mathrm{km}^{2}$ en el estado de Michoacán).

Table 4: Lower physical-geographic unities, integrating the Physical Geographic Regionalization of Michoacan de Ocampo (Identification keys in Plate 1).

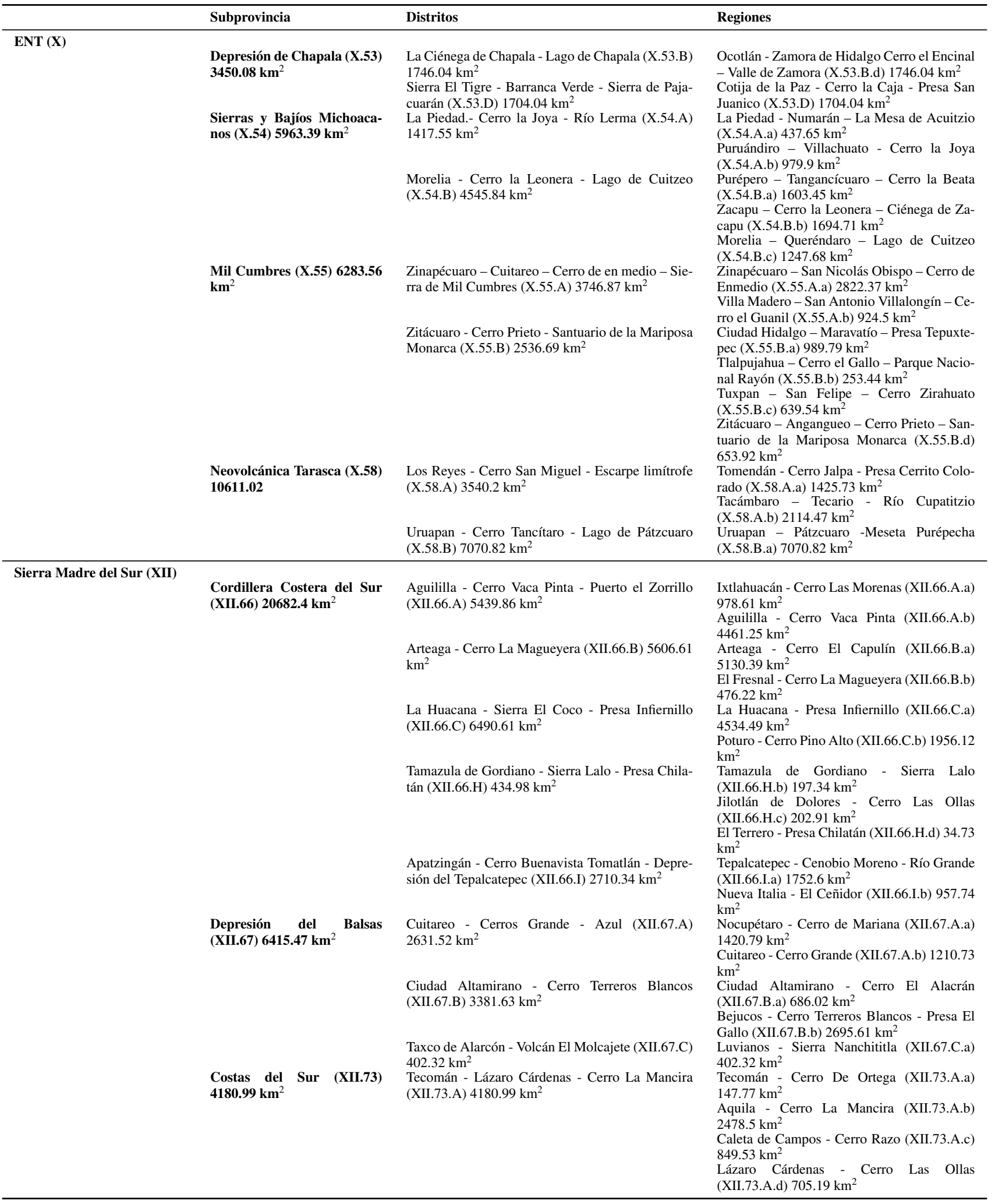




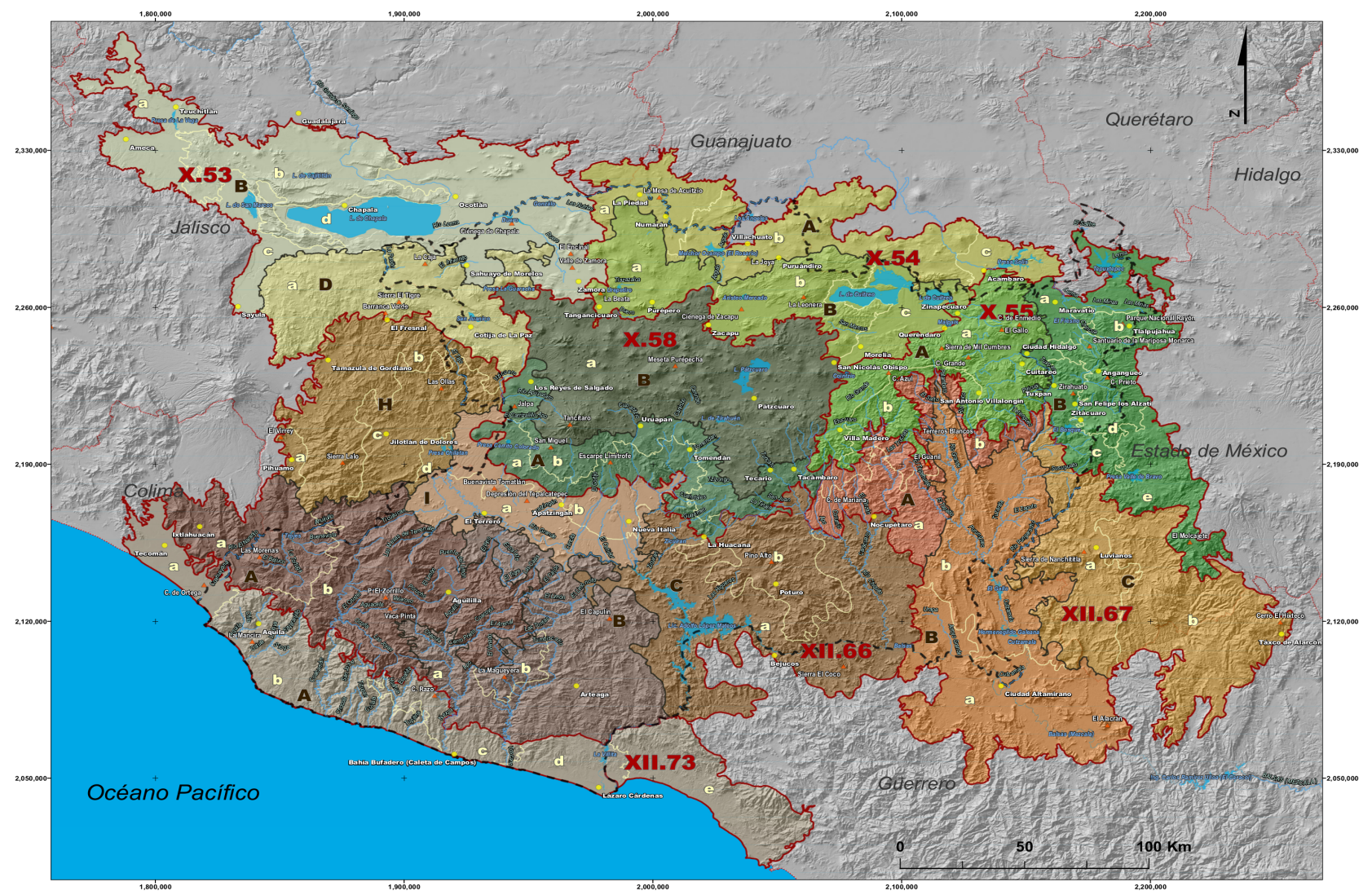

Unidades de paisaje de la RFG de Michoacán

\begin{tabular}{|c|c|c|c|c|}
\hline \multirow{9}{*}{ 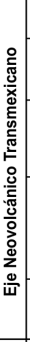 } & $\begin{array}{c}\text { Subprovincias } \\
\text { fisico-geográficas }\end{array}$ & & Distritos físico-geográficos & Regiones físico-geográficas \\
\hline & \multirow{2}{*}{$\begin{array}{l}\text { Depresion de Chapala } \\
\text { X.53 } \\
\end{array}$} & B & La Ciénega - Sahuayo - Lago de Chapala & $\begin{array}{l}\text { (1) Coolan - Zamora de Hidalgo - Cerro el Encinal- } \\
\text { Nvale do Zamora) }\end{array}$ \\
\hline & & D & Sierra El Tigre - Barranca Verde y Sierra La Vainilla & (a) Covja de la Paz - Cerro la Caja - Presa San Juanico \\
\hline & \multirow{2}{*}{$\begin{array}{l}\text { Sierras y Bajios } \\
\text { Michoacanos } \\
\mathbf{X . 5 4}\end{array}$} & & La Piedad - Cerro La Joya - Rio Lerma & 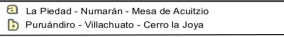 \\
\hline & & B & Morelia - Cerro La Leonera - Lago de Cuitzeo & 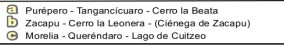 \\
\hline & \multirow[b]{2}{*}{$\begin{array}{l}\text { Mil Cumbres } \\
\mathbf{X . 5 5}\end{array}$} & & Zinapecuaro - Cuitareo - Cerro de en medio - Sierra de Mil Cumbres & 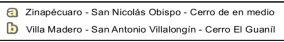 \\
\hline & & & Zitácuaro - Cerro Prieto - Santuario de la Mariposa Monarca & 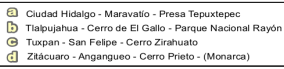 \\
\hline & \multirow{2}{*}{$\begin{array}{c}\text { Neovolcánica Tarasca } \\
\mathbf{X . 5 8} \\
\end{array}$} & $\mathbf{A}$ & Los Reyes - Cerro San Miguel - Escarpe limitrofe & 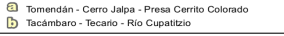 \\
\hline & & B & Uruapan - Cerro Tancitaro - Lago de Pátzcuaro & (a) Unuapan - Pázzcuaro - Meseta Purfopecha \\
\hline \multirow{9}{*}{ 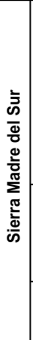 } & \multirow{5}{*}{$\begin{array}{l}\text { Cordillera Costera } \\
\text { del Sur } \\
\text { XII.66 }\end{array}$} & A & Aguililla - Cerro Vaca Pinta - Puerto el Zorrillo & 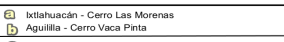 \\
\hline & & B & Arteaga - Cerro La Magueyera & $\begin{array}{l}\text { (enteaga- Cerro EICapulin } \\
\text { B. EI Fresnal - Cerro La Magueyera }\end{array}$ \\
\hline & & c & La Huacana - Sierra El Coco - Presa Infiernillo & $\begin{array}{l}\text { Q La Huacana- - Presa Inferenillo } \\
\text { B. Pouro - Cerro Pino Allo }\end{array}$ \\
\hline & & & Tamazula de Gordiano - Sierra Lalo - Presa Chilatan & 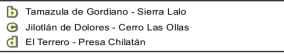 \\
\hline & & $\mathbf{I}$ & Apatzingán - Cerro Buenavista Tomallán - Depresión del Tepalcatepec & 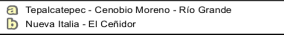 \\
\hline & \multirow{3}{*}{$\begin{array}{l}\text { Depresión del Balsas } \\
\text { XII.67 }\end{array}$} & A & Cultareo - Cerro Grande - Cerro Azul & $\begin{array}{l}\text { Q Nocaupetaro - Cerro de Mariana } \\
\text { Bb Cuttare - Cerro Grande }\end{array}$ \\
\hline & & B & Ciudad Altamirano - Cerro Terreros Blancos & 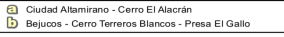 \\
\hline & & c & Taxco de Alarcón - Volcan El Molcajete & (a) Luvianos - Sierra Nanchinula \\
\hline & $\begin{array}{c}\text { Costa del Sur } \\
\mathbf{X I 1 . 7 3} \\
\end{array}$ & A & Lázaro Cárdenas - Tecomán - Cerro La Mancira & 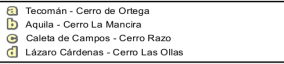 \\
\hline
\end{tabular}

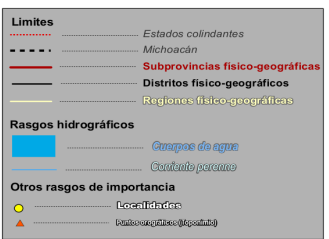

Regiones físico-geográficas externas al límite político-administrativo de Michoacán

\begin{tabular}{|c|c|c|c|}
\hline 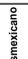 & $\begin{array}{c}\text { Depresion de Chapala } \\
\mathbf{X . 5 3} \\
\end{array}$ & B $\square$ La Ciénega - Sahuayo - Lago de Chapala & 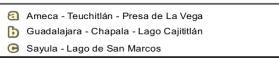 \\
\hline 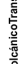 & $\begin{array}{c}\text { Sierras y Bajios } \\
\text { Michoacanos } \\
\text { X.54 } \\
\end{array}$ & A $\square$ La Piedad - Cerro La Joya - Rio Lerma & ๑ Acámbaro - Pressa Solis \\
\hline 旁 & $\begin{array}{l}\text { Mil Cumbres } \\
\mathbf{X . 5 5}\end{array}$ & B $\quad$ Zitácuaro - Cerro Prieto - Santuario de la Mariposa Monarca & ๑ Temascaltepec - Donato Guerra - Presa Valle de Bravo \\
\hline 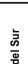 & $\begin{array}{c}\text { Cordillera Costera } \\
\text { del Sur } \\
\text { XII.66 }\end{array}$ & H Tamazula de Gordiano - Sierra Lalo - Presa Chilatan & (1) Pituamo-Cero E Virrey \\
\hline 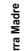 & $\begin{array}{c}\text { Depresión del Balsas } \\
\text { XII.67 }\end{array}$ & c Taxco de Alarcón - Volcan El Molcajete & B. Taxco de Alarón - Cerro EI Huixtoco \\
\hline$\frac{\omega}{\omega}$ & $\begin{array}{l}\text { Costa del Sur } \\
\text { XIII.73 }\end{array}$ & A Lả̇aro Cárdenas - Tecomản - Cerro La Mancira & (๑) San Jose Ixtapa - Cerro La Cruz \\
\hline
\end{tabular}
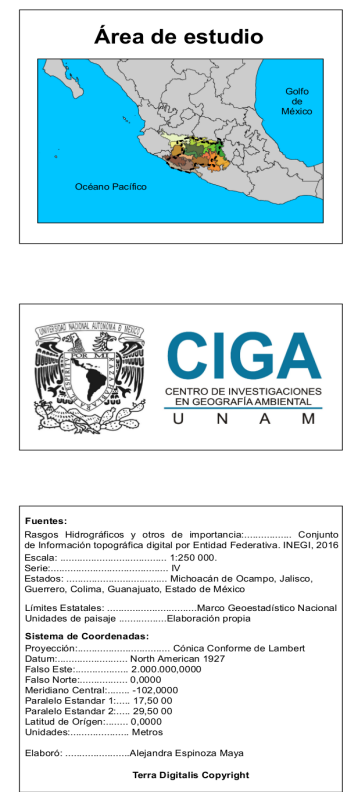

Lámina 1. Mapa de las unidades inferiores de la Regionalización Físico Geográfica de Michoacán de Ocampo. / Plate 1: Map of the lower unities of the Physical Geographic Regionalization of Michoacan de Ocampo. 
Regional. Ello permite entonces, que las Regiones y Distritos fg, puedan ser utilizadas como unidades de gestión ambiental en los ordenamientos ecológicos y territoriales regionales por su homogeneidad y límites precisos, también en los programas de manejo integrado del territorio, en el establecimiento de límites precisos del sistema de Áreas Naturales Protegidas, entre otras aplicaciones. Todo ello hace a la RFG pragmática y utilitaria.

\section{Conclusiones}

La Regionalización Físico Geográfica es un procedimiento de diferenciación y cartografía de unidades de paisaje con un enfoque lógico, sistémico e integrador; los índices diagnóstico que definen cada nivel de la taxonomía, explican de manera clara la diferenciación de individuos físico-geográficos; el enfoque técnico-analítico de la distinción de las unidades espaciales utilizado, estudia el territorio a través de las regularidades de diferenciación espacial, propiedades y dinámica de los paisajes; lo que permitió establecer una nueva diferenciación espacial del territorio de Michoacán.

Los índices diagnóstico aplicados en la distinción de las unidades de paisaje, conforman la guía principal de esta clasificación, definen con claridad los criterios que corresponden a cada nivel en la taxonomía para la identificación de las unidades y permiten el reconocimiento de la interrelación de los elementos naturales que componen los paisajes en cada nivel.

El sistema taxonómico utilizado se forma por unidades espaciales anidadas en siete niveles, permitiendo la cartografía de uno o varios de estos niveles. En el Mapa resultado se cartografían tres niveles taxonómicos que agrupan 36 Regiones, 17 Distritos y 7 Subprovincias físico-geográficas.

En razón de lo expuesto, la aplicación de esta metodología en Michoacán, deja un importante y actual marco de la Geografía Física del territorio, no solo para la descripción y el conocimiento de los elementos que constituyen los componentes naturales y unidades de paisaje en el territorio, sino también para investigaciones posteriores relacionadas con el ordenamiento ambiental, la evaluación, el aprovechamiento sustentable, la restauración o la protección y conservación de los paisajes.

En este tenor, la RFG continuará para el resto del territorio de México, ya que la base de su delimitación (Tipología de los paisajes f-g) está lista para todo el País y los insumos para su actualización se encuentran en diversos inventarios de cartografía digital pertenecientes a las múltiples instituciones encargadas de generar información cartográfica temática y topográfica para México.

\section{Diseño del mapa}

Aunque en el mapa se encuentren representadas las unidades de la RFG al $100 \%$ de su superficie, los porcentajes referidos a los elementos físico-geográficos que las constituyen y las características vinculadas a cada una de las unidades, corresponden únicamente al área que ocupa el Estado de Michoacán, se tomó esa decisión con base en la posible utilización de la RFG en la gestión y planeamiento del territorio, que se fundamenta siempre en límites territoriales de cada Entidad o municipio.

Por otro lado, las unidades externas al límite político administrativo de Michoacán, se muestran únicamente para ilustrar el contexto general, sin embargo no han sido estudiadas a profundidad, ya que el presente trabajo se enfoca particularmente a las unidades dentro de dicho límite.

\section{Software}

El software utilizado es ArcGis 10.1.

\section{Agradecimientos}

Se agradece el apoyo de CONACYT, como la institución que brinda la beca de posgrado (Número de apoyo 362135); a la UNAM, al Posgrado en Geografía y particularmente al CIGA, como el centro donde se realiza la tesis de Doctorado; la colaboración del Dr. Alberto Ortíz Rivera y el Mtro. Rodolfo Ruíz López en la elaboración de la RFG para Guerrero y Jalisco, respectivamente, como precedente para la RFG de Michoacán; el apoyo en campo de la Dra. Sara Barrasa, del Dr. Iván Franch Pardo, de la Mtra. Ayesa Martínez Serano, del Dr. J.M Mateo Rodríguez y de la Lic. Diana Chávez.

\section{Referencias}

Alcorta, R., 1964. Esquema geográfico de México, en Caminos de México. Atlas Goodrich, Euzkadi. Compañía Hulera Euzkadi, México Distrito Federal, pp. 1-2.

Alvarez, M., 1961. Provincias fisiográficas de la República Mexicana. Boletín de la Sociedad Geológica de México 24 (2), 5-20.

Barajas, L., Carrillo, H., Chávez, O., Espinoza, J., Kushida, M., Lacy, R., Lara, A., Méndez, N., Miranda, E., 1986. Regionalización Ecológica del Territorio, en Serie Cuadernos Básicos de Ordenamiento Ambiental. Secretaría de Desarrollo Urbano y Ecología (SEDUE) (4), 1-21.

Berg, L., 1913. Ensayo de la división de Siberia y Turkestán en regiones paisajísticas y morfológicas, en D. N. Sbornik v chest. 70 - letia, Sasnae, Moscú, pp. 167-180, (en ruso).

Bocco, G., Mendoza, M., Velázquez, A., Torres, A., 1999. La regionalización geomorfológica como una alternativa de regionalización ecológica en México. el caso de michoacán de ocampo. Investigaciones Geográficas 40, 7-22.

Bollo, M., Hernández, J., Priego, A., Zaragoza, R., Ortíz, A., Espinoza, A., Ruíz, R., 2015. Una propuesta de Regionalización físico-geográfica de México. Universidad Nacional Autónoma de México (UNAM), Centro de Investigaciones en Geografía Ambiental, (CIGA), Morelia, p. 59.

Cuanalo, C., 1989. Provincias, regiones y subregiones terrestres de México. Colegio de Posgraduados, Chapingo, México, p. 624.

Isachenko, A., 1991. La Geografía del Paisaje y la regionalización físicogeográfica. Educación Superior, Moscú, p. 370, (en ruso).

Mateo, J., 1984. Apuntes de Geografía de los Paisajes,. Imprenta Voisin, p. 470.

Mateo, J., 1989. Regionalización Físico Geográfica de América Central y el Caribe, en Nuevo Atlas Nacional de Cuba. RHEA Consultores - Instituto de Geografía, Cuba - España.

Mateo, J., 2002. Geoecología de los Paisajes: Bases para la Planificación y Gestión Ambiental. Universidad de la Habana. Ministerio de Educación Superior (MES), Cuba, p. 205.

Mateo, J., Da Silva, E., Cavalcanti, A., 2004. Geoecología de los Paisajes. Universidade Federal do Ceará (UFC), Brasil, Fortaleza. 
Ordóñez, E., 1936. Physiographic provinces of Mexico. American Association of Petroleum Geologist 20, 1277-1307.

Ordóñez, E., 1946. Principales Provincias geográficas y geológicas de la República Mexicana. Guía del explorador minero 3, 103-142.

Ortíz, R., Bollo, M., Hernández, S., Priego, S., 2015. La Regionalización físicogeográfica del estado de Guerrero, méxico. Centro de Investigaciones en Geografía Ambiental (CIGA) y Universidad Nacional Autónoma de México (UNAM), Morelia, p. 157.

Quiñones, G., 1987. El sistema fisiográfico de la Dirección General de Geografía. Revista de Geografía 1 (2), 13-20, en Bocco, G., Mendoza, M.; Priego, Á. y Burgos. A, 2009, La cartografía de los sistemas naturales como base geográfica para la planeación territorial. Una revisión bibliográfica. Serie Planeación Territorial. Secretaría de Medio Ambiente y Recursos Naturales (SEMARNAT), Instituto Nacional de Ecología (INE), Universidad Nacional Autónoma de México (UNAM,) Centro de Investigaciones en Geografía Ambiental (CIGA). México, Distrito Federal, 71 pp.

Ramírez-Sánchez, L., Priego-Santander, A., Bollo-Manent, M., 2012. Paisajes Físico-Geográficos del estado de Michoacán. Marco atípico, edición digital, escala 1:250 000. Centro de Investigaciones en Geografía Ambiental (CI-
GA), Universidad Nacional Autónoma de México (UNAM), Campus Morelia, Michoacán.

Secretaría de Desarrollo Urbano y Ecología (SEDUE), 1988. Manual de Ordenamiento Ecológico del Territorio. Subsecretaría de Ecología, Dirección General de Normatividad y Regulación Ecológica, México, Distrito Federal, p. 356.

Suslov-Serguei, P., 1947. Geografía Física de la URSS, Moscú: Siglo XX. Siglo XX, Moscú, (en ruso).

Zaragoza, A., 2010. Tipología y regionalización físico-geográfica de la península de Baja California, México. Tesis profesional de Maestría. Centro de Investigaciones en Geografía Ambiental (CIGA) y Universidad Nacional Autónoma de México (UNAM).

This article accompanies the following material:

HTML: $\quad$ DOI: 10.22201/igg.terradigitalis.2017.1.4.29

Interactive map: DOI: 10.22201/igg.terradigitalis.2017.1.4.14

Static map:
DOI: 10.22201/igg.terradigitalis.2017.1.4.63 